\title{
Angulação de Stent e Implante em Canal Arterial de Neonato com Baixo Peso
}

\author{
Celso Kiyochi Takimura ${ }^{1}$
}

\section{RESUMO}

Relatamos o caso de um neonato portador de cardiopatia congênita cianótica canal-dependente com implante de stent no canal arterial. Uma modificação do stent realizada pelo intervencionista, criando-se um ângulo em seu corpo, possibilitou o avanço do stent da aorta até o canal arterial, e seu subsequente implante nesse sítio.

DESCRITORES: Recém-nascido. Cardiopatias congênitas. Stents. Canal arterial.

$\mathbf{N}$ eonatos portadores de cardiopatia congênita cianótica canal-dependente requerem, além de tratamento medicamentoso (infusão de prostaglandina E1), medidas para aumentar o fluxo pulmonar, como a realização cirúrgica de shunt sistêmico-pulmonar (Blalock-Taussig).

A morbidade e a mortalidade desse procedimento cirúrgico podem ser altas e, em alguns casos, o implante de stent no canal arterial é uma boa alternativa ao shunt cirúrgico sistêmico-pulmonar. A morfologia do canal arterial em cardiopatias congênitas cianóticas tem grande variabilidade, podendo originar-se mais proximalmente no arco aórtico, tornando difícil, ou mesmo impossível, o avanço do stent da aorta até o canal arterial por meio de técnicas percutâneas habituais. ${ }^{1}$

Relatamos, no presente caso clínico, uma modificação do stent realizada pelo intervencionista, com a realização de uma angulação em seu corpo, a qual possibilitou o avanço do stent da aorta até o canal arterial e seu implante em um neonato de baixo peso, portador de atresia pulmonar com comunicação interventricular e contraindicação para a cirurgia de Blalock-Taussig.

\section{ABSTRACT}

Stent Angulation and Implantation in the Ductus Arteriosus of a Low-Weight Neonate

We report the case of a neonate with ductus-dependent cyanotic congenital heart disease treated by stenting in the ductus arteriosus. A physician-modified stent angulation enabled the advance of the stent from the aorta to the ductus arteriosus and its subsequent implantation.

DESCRIPTORS: Infant, newborn. Heart defects, congenital. Stents. Ductus arteriosus.

\section{RELATO DO CASO}

Neonato do sexo feminino, nascido com 2,1 kg, a termo, de parto natural, sem diagnóstico pré-natal de cardiopatia, apresentou, logo ao nascer, cianose central (saturação de oxigênio de $80 \%$ ), ausculta de sopro cardíaco sistólico em bordo esternal esquerdo ++/4+, segunda bulha única e pulsos periféricos normais.

Ecocardiograma transtorácico revelou a presença de atresia pulmonar com comunicação interventricular e canal arterial patente (diâmetro de $3 \mathrm{~mm}$ ). Foram realizados entubação endotraqueal, assistência ventilatória e início de administração endovenosa de prostaglandina E1.

O neonato evoluiu com níveis de saturação de oxigênio periférico entre 80 e 90\%, insuficiência renal (pico de creatinina sérica de até $2,3 \mathrm{mg} / \mathrm{dL}$ ), plaquetopenia (40.000 plaquetas $/ \mathrm{mm}^{3}$ ), hemorragia pulmonar e pequena hemorragia cerebral. Foi avaliado pela equipe de cirurgia cardíaca, que contraindicou a realização de shunt sistêmico-pulmonar, pelo alto risco cirúrgico (baixo peso, insuficiência renal e plaquetopenia), e indicou implante de stent no canal arterial, para paliação inicial.
N.E.: As imagens angiográficas deste Relato de Caso foram obtidas diretamente da tela do monitor do equipamento de fluoroscopia.

${ }^{1}$ Doutor. Médico assistente no Hospital da Luz, São Paulo, SP, Brasil.
Correspondência: Celso Kiyochi Takimura. Avenida Açocê, 92, apto. 162 - Indianópolis - São Paulo, SP, Brasil - CEP 04075-020

E-mail: celso.takimura@incor.usp.br

Recebido em: 22/11/2012 • Aceito em: 29/10/2013 
Após melhora clínica da criança, no $15^{\circ}$ dia de vida, foi suspensa a infusão endovenosa de prostaglandina E1 2 horas antes do início do procedimento hemodinâmico, com o intuito de reduzir o tamanho do canal arterial e facilitar o implante do stent.

Sob anestesia geral, foram realizadas punção da artéria femoral direita e inserção de introdutor valvulado $4 \mathrm{~F}$ de $11 \mathrm{~cm}$ (Cordis Corporation, NJ, Estados Unidos). Sob fluoroscopia, realizou-se aortografia torácica com cateter Judkins Right 4 F (Cordis Corporation, NJ), Estados Unidos), o qual demonstrou canal arterial patente, originando-se do arco aórtico, com diâmetro aproximado de $3 \mathrm{~mm}$ e trajeto vertical (Figura 1). Não visualizamos, nessa aortografia, fístulas aorto-pulmonares.

A seguir, com um cateter Pigtail 4 F (Cordis Corporation, NJ, Estados Unidos) cortado em sua extremidade distal, de modo a se obter um cateter tipo "J invertido", foi cateterizado seletivamente o canal arterial. Introduziu-se, por esse cateter, um fio-guia de 0,014 polegadas Runthrough $^{\circledR}$ NS (Terumo Corporation, Tóquio, Japão) até o canal arterial, tronco pulmonar e periferia do leito arterial pulmonar. Sem a utilização de cateter-guia diagnóstico ou terapêutico, foi introduzido, por sobre o fio-guia de 0,014 polegadas, um stent coronário Multi-Link Vision ${ }^{\circledR}$ (Abbott Vascular, CA, Estados Unidos) $3,5 \times 15 \mathrm{~mm}$. Entretanto, essa primeira tentativa de implante não teve sucesso por causa do ângulo de $90^{\circ}$ de entrada no canal arterial em relação à aorta, que fez com que o stent se direcionasse para a aorta ascendente. Optamos pela realização de uma angulação de $45^{\circ}$ na porção média do stent (Figura 2), com o

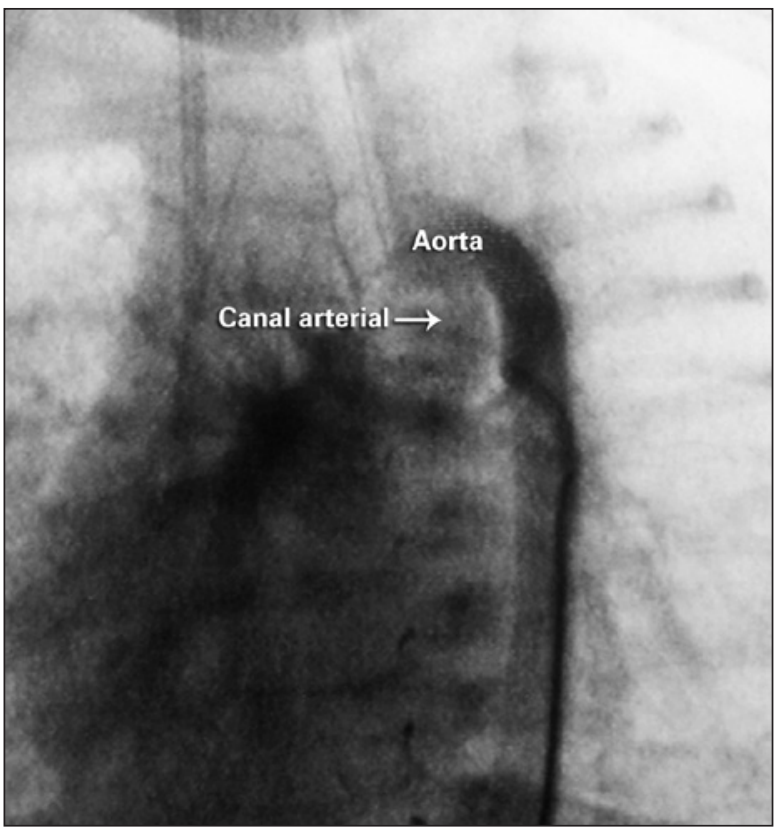

Figura 1. Aortografia revelando canal arterial "vertical" (seta) originando-se proximalmente do arco aórtico. intuito de facilitar a passagem do stent da aorta para o canal arterial. O stent coronário com essa angulação foi reintroduzido por sobre o fio-guia e avançou da aorta até o canal arterial sem resistência, sem o uso de cateter-guia ou introdutor valvulado longo; esse stent foi implantado no canal arterial com pressão de liberação de 20 atm. A aortografia ao final do procedimento revelou stent bem posicionado e expandido no canal arterial (Figura 3). Administramos $20 \mathrm{~mL}$ de concentrado de plaquetas e retiramos o introdutor valvulado arterial sem intercorrências. A criança retornou para a Unidade de Terapia Intensiva Pediátrica em condições clínicas estáveis, com saturação arterial de oxigênio de $98 \%$.

\section{DISCUSSÃO}

Neonatos com cardiopatia congênita cianótica canal-dependente em geral se apresentam com quadros clínicos graves e complexos, ainda representando um grande desafio diagnóstico e terapêutico. ${ }^{2}$

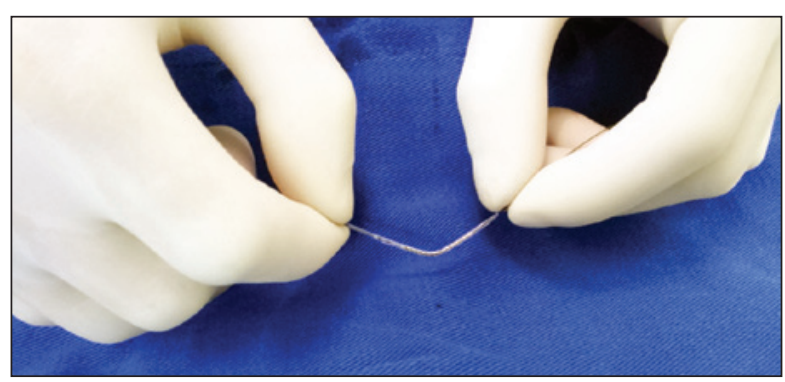

Figura 2. Modificação do stent, com realização de angulação de $45^{\circ}$ no corpo do stent.

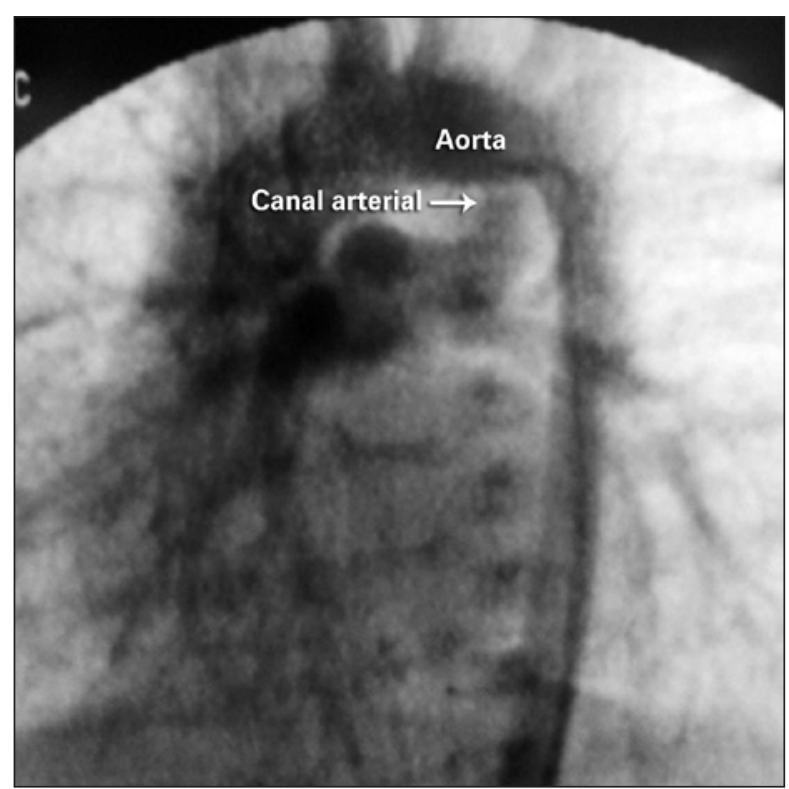

Figura 3. Aortografia revelando stent implantado e bem expandido no canal arterial (seta). 
O implante de stent no canal arterial foi descrito inicialmente em 1992 por Gibbs et al., ${ }^{3,4}$ com resultados iniciais pouco animadores. Desde então, vários grupos publicaram sua experiência, com detalhamento da técnica de implante de stent no canal arterial. ${ }^{1,5,6}$

Alguns excluem pacientes com menos de $2,5 \mathrm{~kg}$, pelo risco de dano grave às artérias femorais com $\mathrm{O}$ uso de introdutores valvulados $4 \mathrm{~F}$ longos. ${ }^{1}$ Para os casos em que o canal arterial origina-se mais proximalmente no arco aórtico, como na atresia pulmonar com comunicação interventricular, esse mesmo grupo tem a via venosa como via preferencial de implante de stent no canal arterial. Ressaltam, entretanto, o risco do desenvolvimento de bloqueio atrioventricular total, à medida que o cateter fricciona as bordas da comunicação interventricular durante a manipulação do mesmo.

Para os casos com alto risco cirúrgico de se submeterem à realização de um shunt sistêmico-pulmonar, como no presente caso, o implante de stent no canal arterial é uma boa medida paliativa inicial. A modificação do stent, criando-se uma angulação de $45^{\circ}$ em seu corpo, foi, neste caso, determinante para o implante do stent no canal arterial, cuja origem proximal do arco aórtico e trajeto peculiar tornaram esse canal também conhecido pelo nome de canal arterial "vertical". Esse grau de angulação no stent não causou dano estrutural e nem modificação de seu comportamento à insuflação ou distorção no local implantado detectáveis clinicamente.

Não foi encontrado, na literatura médica pesquisada, relato prévio da realização de angulação em stent coronário, facilitando sua implantação em canal arterial de neonato com baixo peso com cardiopatia congênita cianótica canal-dependente. Até o momento da redação deste relato de caso, outros três neonatos com cardiopatia congênita canal-dependente foram submetidos com sucesso ao implante de stent no canal arterial por essa técnica descrita.

\section{CONFLITO DE INTERESSES}

Os autores declaram não haver conflito de interesses relacionados a este manuscrito.

\section{REFERÊNCIAS}

1. Alwi M. Stenting the ductus arteriosus: case selection, technique and possible complications. Ann Pediatr Card. 2008;1(1):38-45.

2. Silva CMC, Gomes LFG. Reconhecimento clínico das cardiopatias congênitas. Rev Soc Cardiol Estado de São Paulo. 2002;12(5):717-23.

3. Gibbs J, Rothman M, Rees M, Parsons J, Blackburn M, Ruiz C. Stenting of the arterial duct: a new approach to palliation for pulmonar atresia. Br Heart J. 1992;67(3):240-5.

4. Gibbs JL, Uzun O, Blackburn MEC, Wren C, Hamilton L, Watterson KG. Fate of the stented arterial duct. Circulation. 1999;99(20):2621-5.

5. Alwi M, Choo KK, Latiff HA, Kandavello G, Samion H, Mulyadi MD. Initial results and medium-term follow-up of stent implantation of patent ductus arteriosus in duct-dependent pulmonary circulation. J Am Coll Cardiol. 2004;44(2):438-45.

6. Matter M, Almarsafawey H, Hafez M, Attia G, Abuelkheir M. Patent ductus arteriosus stenting in complex congenital heart disease: early and midterm results for a single-center experience at Children Hospital, Mansoura, Egypt. Pediatr Cardiol. 2013; 34(5):1100-6. 